\title{
Methodology for Digital Money based on General Cryptographic Tools ${ }^{\star}$
}

\author{
Stefano D'Amiano ${ }^{1}$ and Giovanni Di Crescenzo ${ }^{2}$ \\ ${ }^{1}$ Computer Science Department, \\ Cornell University, Ithaca, NY, USA \\ 2 Dipartimento di Informatica ed Applicazioni, \\ Università di Salerno, 84081 Baronissi (SA), Italy
}

\begin{abstract}
In this paper we investigate methodologies for off-line digital cash using general cryptographic tools. First we give a way for off-line spending of coins using non-interactive zero-knowledge proofs of knowledge with preprocessing. Under this paradigm and using other general cryptographic tools, we show how to obtain the property of dividability of coins and give a technique for avoiding double spending of coins. Chaum and Pedersen considered a model in which the Bank discovers the anthor of a double spending of a coin immediately after that coin has been deposited, and proved that in this model transferred coins grow in size. We consider a different model and show how to obtain transferability of coins without any increase in size.
\end{abstract}

\section{Introduction}

An electronic cash system is the digital equivalent of paper cash and can be seen as a collection of protocols with one distinguished player called Bank. It usually supports transactions of four different types, each one being the digital equivalent of a real-life transaction: opening an account, withdrawing, spending and depositing a coin.

An electronic cash system has the property of off-line spending of coins if the Bank is not involved in the protocol for spending a coin. The main approaches to the construction of an electronic cash system with the property of off-line spending are three and are based respectively on blind signatures, on zero-knowledge proofs of knowledge and on oblivious authentication.

A blind signature scheme is a protocol between two parties in which a first party (in a cash system, the Bank) signs a message received by the second party (in a cash system, a user). The signature given is blind in the sense that the first party does not get useful informations about the message signed. The first blind signature scheme has been given in [4] using RSA. Other electronic cash systems based on blind signatures are in $[3,12]$.

* Partially supported by Italian Ministry of University and Research (M.U.R.S.T.) and by National Council for Research (C.N.R.). 
An oblivious authentication scheme is a protocol between two parties in which a first party (in a cash system, the Bank) issues to a second party (in a cash system, a user) a digital document in which the user's identity is embedded in some way, together with a witness of this identity. The authentication given is oblivious in the sense that the first party is not able at a later stage to link the digital document to the time in which he has issued it. An oblivious authentication scheme has been given in [13] where it was implemented using one-way functions plus a preprocessing stage and using RSA.

A non-interactive zero-knowledge proof system of knowledge with preprocessing is a protocol between two parties in which, after an interactive preprocessing, the first party (in a cash system, the spender) can send a single message to the second party (in a cash system, the receiver) such that this message represents a zero-knowledge proof (see [15]) that the first party knows a witness for the truth of a certain statement. Such a system can be used by a spender to prove to the receiver the knowledge of a signature of a coin released by the Bank. A non-interactive zero-knowledge proof system of knowledge with preprocessing based on general complexity assumptions and a cash system based on it have been given in [6] (see also [21]).

\section{Our results.}

Taking the paper [6] as a starting point, we investigate methodologies for off-line digital cash using general cryptographic tools. First we give a general way for offline spending of coins using non-interactive zero-knowledge proofs of knowledge with preprocessing. Under this paradigm and using other general cryptographic tools, we show how to obtain the property of dividability of coins and give a technique for avoiding multiple spending of coins even when the coin is transferred to many users before being deposited to the Bank.

In [5] it is showed that transferred cash grows in size. The model considered requires that the Bank discovers the author of a double spending of a coin immediately after that coin has been deposited. By considering a different model, we show how to obtain transferability of coins without any increase in their size.

\section{Organization of the paper.}

In Section 2 we give some notations and review some background cryptographic notions that will be useful in our construction. In Section 3 we recall definition and properties of electronic cash systems. In Section 4 we give a general way for off-line spending of coins using non-interactive zero-knowledge proofs of knowledge with preprocessing. In Section 5 we show how to obtain the property of dividability of coins. In Section 6 we give a technique for avoiding multiple spending of coins. In Section 7 we give a sketch of proof of the correctness of our construction.

\section{Notations and basic tools}

Notations. We use the symbols o and $\oplus$ to denote respectively concatenation and bitwise xor of binary strings. If $S$ is a probability space, then " $x-S$ " de- 
notes the algorithm which assigns to $x$ an element randomly selected according to $S$. If $p(\cdot, \cdot, \cdots)$ is a predicate, the notation $\operatorname{Prob}(x \leftarrow S ; y \leftarrow T ; \ldots: p(x, y, \cdots))$ denotes the probability that $p(x, y, \cdots)$ will be true after the ordered execution of the algorithms $x \leftarrow S, y \leftarrow T, \ldots$. The notation $\{x \leftarrow S ; y \leftarrow T ; \cdots:(x, y, \cdots)\}$ denotes the probability space over $\{(x, y, \cdots)\}$ generated by the ordered execution of the algorithms $x \leftarrow S, y \leftarrow T, \cdots$ By the writing $(\mathrm{A}, \mathrm{B})$ we denote a pair of Turing machines $\mathrm{A}$ and $\mathrm{B}$. By the writing $\left(o_{a}, o_{b}\right)=(\mathrm{A}, \mathrm{B})\left(i_{a}, i_{b}\right)$ we denote an execution of the protocol $(\mathrm{A}, \mathrm{B})$, where A's input is $i_{a}, \mathrm{~B}$ 's input is $i_{b}$, A's output is $o_{a}$ and B's output is $o_{b}$.

\section{Basic tools.}

Now we briefly review the concepts of pseudo-random functions, secure commitment schemes, signature schemes and non-interactive zero-knowledge proofs of knowledge with preprocessing, all playing an important role in our construction.

Pseudo-random Functions. The concept of pseudo-random function has been introduced by Goldreich, Goldwasser, and Micali [14]. Intuitively, we can say that a collection $F=\left\{f_{s}\right\}$ of functions $f_{s}:\{0,1\}^{n} \rightarrow\{0,1\}^{n^{c}}$ is $c$-pseudo-random if the output of a function randomly chosen from the collection and evaluated on arguments chosen by a polynomial time algorithm cannot be distinguished from the output of a truly random function. In [14] it is shown how to construct a $c$ pseudo-random collection of function for any constant $c$ from any pseudo-random generator. In the sequel, whenever the constant $c$ is clear from the contest, we will just say pseudo-random function.

Secure Commitment Schemes. Given the coin tosses $s, r$ and a bit $b$, a secure commitment scheme for the bit $b$ is an efficient algorithm $E$ such that: 1) it is hard to compute a commitment which can be decommitted both as 0 and as 1 , and 2) it is computationally hard to distinguish commitments of 0 from commitments of 1 , where the string $d=E(b, s, r)$ is a commitment to bit $b$, and the strings $s, r$ are a decommitment of $d$ as the bit $b$. The secure commitment scheme can be easily extended to any string $x$. If $x=b_{1} \ldots b_{m}$ is an $m$-bit string then $E(x, s, r)$ is intended to consist of the $m$ different commitments $E\left(b_{i}, s, r_{i}\right)$, $i=1, \ldots, m$, where $r=r_{1} \ldots r_{m}$ is an $n m$-bit string. In this case, it can be seen that property 2 can be extended to any two strings. Also, we observe that if $d=E(x, s, r)$ and $s$ and $r$ are known, then the string $x$ committed to by $d$ can be efficiently computed. This definition of commitment is inspired by the bitcommitment protocol of Naor [18] which is based on any pseudorandom number generator. The commitment is based on a random challenge given to the committer. For a random challenge and a random generator, there is no way to cheat in a commit phase. Whenever a random reference string $\sigma$ is available, we can implement the commitment scheme by applying Naor's protocol by choosing the generator (one way function) and the long-enough challenging string non-interactively from some portion of the random string $\sigma$ (used just for this purpose). All the commitments will, then, use this choice (for more details on this, see [18]). 
Signature Schemes. A signature scheme is a triple $(G, S, V)$ of efficient algorithms. $G$ is a generation algorithm that on input a random string outputs a pair $(p k, s k)$, where $p k$ is a public key and $s k$ is a secret key. $S$ is a signing algorithm that on input a message $m$ and the secret key $s k$, outputs a signature $s i g_{m} . V$ is a verification algorithm that on input a message $m$, the public key $p k$ and the signature $s i g_{m}$, verifies that $s i g_{m}$ is a signature of message $m$ computed using the signing algorithm $S$. Two important properties of signature schemes are that any party can verify the correctness of a signature of a given document, and that no party can forge a signature for a new document, even after seeing arbitrary signature samples adaptively chosen. Signature schemes have been introduced by [10] and further elaborated by [16], [17], [19] and [22].

Zero-knowledge Proofs of Knowledge. Zero-knowledge proofs of membership were introduced in [15]. Roughly speaking, a zero-knowledge proof of membership is a two-party protocol in which one party, called the Prover, convinces the second party, called the Verifier, that a certain input string $x$ belongs to a language $L$ without releasing any additional knowledge. Subsequently, in [23] and [11] the concept of a zero-knowledge proof of knowledge was considered. Here, the Prover wants to convince the Verifier that he knows a witness $w$ such that the polynomial-time relation $R(x, w)$ holds.

Non-Interactive Zero-Knowledge Proofs of Knowledge with Preprocessing. The concept of a non-interactive zero-knowledge (NIZK) proof has been introduced by [2] where it is proved that, if a random string readable by both the prover and the verifier is available, it is possible for the prover to give noninteractive zero-knowledge proofs for any NP language. (See [1] for formal definitions and proofs.) The definition of a NIZK proof of knowledge has been given in [7] where it is shown a NIZK proof of knowledge for any polynomial-time relation.

In a NIZK proof of knowledge with preprocessing, after an interactive preprocessing stage, a prover can give any polynomial (in the length of the preprocessing stage) number of NIZK proofs of knowledge to the verifier. Let $R$ be a polynomial-time relation and $L_{R}$ be the language of strings $x$ such that there exists $w$ for which $R(x, w)=1$. Here we recall the definition of [6] of NIZK proof system of knowledge with preprocessing for the relation $R$.

Definition 1. Let $P=\left(P_{1}, P_{2}\right)$ and $V=\left(V_{1}, V_{2}\right)$, where $P_{1}$ and $V_{1}$ are interactive probabilistic poly-time Turing machines, $\mathrm{P}_{2}$ is a probabilistic poly-time Turing machine, and $\mathrm{V}_{2}$ is a deterministic poly-time Turing machine. The pair $(\mathrm{P}, \mathrm{V})$ is a Non-Interactive Zero-Knowledge Proof System of Knowledge with Preprocessing for the polynomial-time relation $R$ if:

1. Completeness: For all constants $c$, all $(x, w) \in R$ and all sufficiently large $n$,

$$
\begin{gathered}
\operatorname{Prob}\left((\alpha, \beta) \leftarrow\left(\mathrm{P}_{1}, \mathrm{~V}_{1}\right)\left(1^{n}\right) ;(x, \text { Proof }) \leftarrow \mathrm{P}_{2}(\alpha, x, w):\right. \\
\left.\mathrm{V}_{2}(\beta, x, \text { Proof })=1\right) \geq 1-1 / n^{c} .
\end{gathered}
$$


2. Soundness: There exists an extractor algorithm $\mathrm{E}=\left(\mathrm{E}_{1}, \mathrm{E}_{2}\right)$ such that for all pairs $\mathrm{P}^{\prime}=\left(\mathrm{P}_{1}^{\prime}, \mathrm{P}_{2}^{\prime}\right)$ of efficient algorithms, for all $d$ and all sufficiently large $n$,

$$
\begin{gathered}
\operatorname{Prob}\left((\alpha, \beta) \leftarrow\left(\mathrm{P}_{1}^{\prime}, \mathrm{E}_{1}\right)\left(1^{n}\right) ;(x, \text { Proof }) \leftarrow \mathrm{P}_{2}^{\prime}(\alpha) ;\right. \\
\left.w \leftarrow \mathrm{E}_{2}(\beta, x, \operatorname{Proof}):(x, w) \in R\right)>\left(1-n^{-d}\right) . \\
\operatorname{Prob}\left((\alpha, \beta) \leftarrow\left(\mathrm{P}_{1}^{\prime}, \mathrm{V}_{1}\right)\left(1^{n}\right) ;(x, \operatorname{Proof}) \leftarrow \mathrm{P}_{2}^{\prime}(\alpha): \mathrm{V}_{2}(\beta, x, \operatorname{Proof})=1\right) .
\end{gathered}
$$

3. Zero-Knowledge: For each $\mathrm{V}^{\prime}$, there exists an efficient algorithm $\mathrm{S}$ such that for all $x_{1}, x_{2}, \ldots \in L_{R}$, for all efficient nonuniform algorithms $D$, for all constants $e$, and all sufficiently large $n$,

$$
\begin{aligned}
& \mid \operatorname{Prob}\left(y \leftarrow \operatorname{View}_{\mathrm{V}^{\prime}}\left(1^{n}, x_{1}, x_{2}, \cdots\right): D_{1^{n}}(y)=1\right)- \\
& \operatorname{Prob}\left(y \leftarrow S\left(1^{n}, x_{1}, x_{2}, \cdots\right): D_{1^{n}}(y)=1\right) \mid<n^{-e},
\end{aligned}
$$

where View $V^{\prime}\left(1^{n}, x_{1}, x_{2}, \cdots\right)$ is the view of $V^{\prime}$ in the executions of $\left(\mathrm{P}_{1}, \mathrm{~V}_{1}\right)$ and $\left(\mathrm{P}_{2}, \mathrm{~V}_{2}\right)$ on inputs $x_{1}, x_{2}, \ldots$

Essentially, we can think of $\left(\mathrm{P}_{1}, \mathrm{~V}_{1}\right)$ as the protocol for the preprocessing stage and of $\left(\mathrm{P}_{2}, \mathrm{~V}_{2}\right)$ as the (non-interactive) protocol for the proof stage.

A NIZK proof of knowledge with preprocessing has been given in [6]. An anonymous version of this protocol is in [9], where a verifier, after executing many preprocessing protocols, is not able to associate the conversation of a proof protocol to any preprocessing protocol. We will use this version in the construction of our electronic cash system.

\section{Electronic cash systems}

An Electronic Cash System is the digital counterpart of paper cash. It can be seen as a set of cryptographic protocols where the players are a Bank $B$ and a collection of users $\left\{U_{i}\right\}$.

An electronic cash system should allow the supportability of transactions and operations representing the digital equivalent of (at least) all the real-life operations, as opening an account; withdrawing, depositing and spending a coin. Also, it can have some additional properties, as dividability of coins and off-line spending of coins or any desired piece of coins. On the other hand, as real-life coins are physical objects, they guarantee a satisfactory level of security to a bank and to the users. Then an electronic cash system should provide (at least) the same level of security guaranteed by its physical counterpart.

Our definition of electronic cash system is essentially based on those of $[4,13]$.

Definition 2. An off-line electronic cash system is a pair $(\mathcal{S}, \mathcal{Q})$, where $\mathcal{S}=$ $\left\{B a n k, U_{1}, \ldots, U_{n}\right\}$ is a set of interactive probabilistic polynomial-time Turing machines, and $\mathcal{Q}$ is the fourtuple (OPEn, Withdraw, SPEnd, Deposit). OPEN $=\mathrm{O}=\left(\mathrm{O}_{1}, \mathrm{O}_{2}\right)$ is a protocol executed by the Bank and a user $U_{i}$ and allows user $U_{i}$ to open an account; WITHDRAW $=\mathrm{W}=\left(\mathrm{W}_{1}, \mathrm{~W}_{2}\right)$ is a protocol executed by 
the Bank and a user $U_{i}$ and allows user $U_{i}$ to withdraw a coin from his account; SPEND $=\mathrm{S}=\left(\mathbf{S}_{1}, \mathbf{S}_{2}\right)$ is a protocol executed by two users $U_{i}$ and $U_{j}$ and allows user $U_{i}$ to pass a coin to user $U_{j} ;$ DEPOSIT $=\mathrm{D}=\left(\mathrm{D}_{1}, \mathrm{D}_{2}\right)$ is a protocol executed by the Bank and a user $U_{i}$ and allows user $U_{i}$ to deposit a coin into his account. Moreover, the pair $(\mathcal{S}, \mathcal{Q})$ satisfies the following requirements:

1. No forging: For all integers $k>0$, given the transcripts of $k$ protocols $\mathbf{W}_{1}, \ldots, \mathbf{W}_{k}$, for each efficient nonuniform algorithm $A d v$, the probability that Adv computes $k+1$ coins $c_{1}, \ldots, c_{k+1}$ such that for each $i=1, \ldots, k+1$, $\mathrm{S}=(\operatorname{Adv}, \cdot)\left(c_{i}, \cdot\right)=(\cdot$, accept $)$ is negligible in $n$.

2. No tracing: For all integers $k>0$, given the transcripts of $k$ protocols $\mathrm{W}_{1}, \ldots, \mathrm{W}_{k}$ and of $k$ protocols $\mathrm{D}_{1}, \ldots, \mathrm{D}_{k}$, for each efficient nonuniform algorithm $A d v$, for each coin $c$ such that $\mathrm{D}=(\cdot, A d v)(c, \cdot)=(\cdot$, accept $)$ with nonnegligible probability, for each $i, j \in\{1, \ldots, n\}$, the probability that Adv computes $l \in\{i, j\}$ such that $\mathrm{W}=\left(\operatorname{Adv}, \mathrm{U}_{l}\right)\left(\cdot, c_{i}\right)=(\cdot, a c c e p t)$ or $\mathrm{S}=\left(\mathrm{U}_{l}, \cdot\right)\left(c_{i}, \cdot\right)=(\cdot, a c c e p t)$ is at most $1 / 2+$ a term negligible in $n$.

3. No double spending: For each efficient nonuniform algorithm $A d v$, for each pair $\left(c_{1}, c_{2}\right)$ of coins such that $\mathrm{S}=\left(\operatorname{Adv}, \mathrm{S}_{i}\right)\left(c_{i}, \cdot\right)=(\cdot$, accept $)$ for $i=1,2$ with nonnegligible probability, there exists an efficient algorithm Detect such that, given the transcripts of the execution of protocol $\mathrm{S}=\left(A d v, \mathrm{~S}_{i}\right)$ on input $\left(c_{i}, \cdot\right)$, for $i=1,2$, outputs $I D_{A d v}$ with nonnegligible probability in $n$.

4. No framing: For all integers $k>0$, given the transcripts of $k$ protocols $\mathrm{W}_{1}, \ldots, \mathrm{W}_{k}$ and of $k$ protocols $\mathrm{D}_{1}, \ldots, \mathrm{D}_{k}$, for each efficient nonuniform algo$\operatorname{rithm} A d v$, for each coin $c$, for each $i, j \in\{1, \ldots, n\}$, the probability that $A d v$ computes $l \in\{i, j\}$, and $c_{1}, c_{2}$ such that $\mathrm{S}=\left(\mathrm{U}_{l}, \mathrm{U}_{h}\right)\left(c_{1} \cdot\right)=\left(\cdot, c_{1}\right.$ o accept $)$ and $\mathrm{S}=\left(\mathrm{U}_{l}, \mathrm{U}_{k}\right)(c, \cdot)=\left(\cdot, c_{2} \circ\right.$ accept $)$, for some $h, k \in\{1, \ldots, n\}$ is at most $1 / 2+$ a term negligible in $n$.

Instead of giving a complete description of protocols OPEN, WITHDRAW, SPEND, DEPOSIT, which would be hard to read, we divide the presentation into three parts. First we give a way for off-line spending of coins using non-interactive zero-knowledge proofs of knowledge with preprocessing. Then, using other general cryptographic tools as pseudo-random functions and secure commitment schemes, we show how to obtain dividability of coins. Finally, we give a technique based on signature schemes for avoiding multiple spending of coins even when the coin is transferred to many users before being deposited to the Bank.

\section{NIZK proofs of knowledge and digital money}

In this section we show how non-interactive zero-knowledge proofs of knowledge with preprocessing together with secure commitment schemes and signature schemes give a methodology for obtaining off-line spendable digital money. 


\subsection{The cash system}

Let $E(\cdot, \cdot, \cdot)$ be a secure commitment scheme and let $\left(p k_{B}, s k_{B}\right)$ be a pair of a public and a secret key specifying the signature scheme $\left(G_{B}, S_{B}, V_{B}\right)$ of the Bank. Also, let $(\mathrm{P}, \mathrm{V})$ be the NIZK proof of knowledge with preprocessing for any NP-complete language given in [9]; we denote by $\left(\mathrm{P}_{1}, \mathrm{~V}_{1}\right)$ its preprocessing protocol and by $\left(\mathrm{P}_{2}, \mathrm{~V}_{2}\right)$ its proof protocol. Finally, let $n$ be a security parameter, $\sigma$ be a sufficiently long random reference string, and $I D_{B}, I D_{U}$ be $n$-bit strings denoting the identity of the Bank and of user $U$ respectively.

We assume that at the beginning of the cash system all the above tools are written on a public file PF. Then, in the protocol of the opening of an account, a user $U$ and the Bank establish some common information which will allow user $U$ to give non-interactive zero-knowledge proofs of knowledge at a later stage. Essentially, they run the preprocessing stage of the given proof system of knowledge.

\section{Opening the account:}

- Bank and U: run the protocol $\left(P_{1}, V_{1}\right)$, where $U$ runs algorithm $P_{1}$ and the Bank runs algorithm $V_{1}$.

The protocol for withdrawing a coin is made of two rounds: first the user $U$ sends its request to the Bank, and then the Bank sends its authorization to user $U$. Informally, the protocol is the following: the user $U$ randomly generates a string $c$ and sends to the Bank a commitment to $c$ computed using the secure commitment scheme $E$. The Bank answers to $U$ with a signature of the commitment, computed using her signature scheme $\left(G_{B}, S_{B}, V_{B}\right)$. The knowledge of this signature will represent her authorization to spend the coin $c$. More formally, the protocol for withdrawing a coin is the following:

\section{Withdrawing a coin:}

- U: randomly choose an $n$-bit string $c$ and an $n^{2}$-bit string $r$; compute com $=E(c, \sigma, r)$ and send com to the Bank.

- Bank: compute $s i g_{c o m}=S_{B}\left(s k_{B}, c o m\right)$ and send $s i g_{c o m}$ to $U$.

- U: if $V_{B}\left(p k_{B}, c o m, s i g_{c o m}\right)=1$ then accept the coin $c$.

Observe that the commitment to $c$ is necessary, for otherwise the coin $c$ would be later easily traceable from the Bank.

The protocol for spending a coin uses the non-interactive proof of knowledge. To pass a coin $c$ to user $U_{2}$, user $U_{1}$ sends him the string $c$ and a non-interactive zero-knowledge proof of knowledge of a commitment to $c$ and of a signature of this commitment released by the Bank. If the proof is convincing, then user $U_{2}$ accepts the coin $c$ from $U_{1}$ : More formally, the protocol for spending a coin is the following: 


\section{Spending a coin:}

- $\mathrm{U}_{1}$ : let $T$ be the statement "there exist $r, c o m, \operatorname{sig}_{\text {com }}$ s. t. com $=E(c, \sigma, r)$ and $V_{B}\left(p k_{B}, c o m, s i g_{c o m}\right)=1 "$; use algorithm $\mathrm{P}_{2}$, inputs $r$, com, sig com $_{\text {, }}$, and $\sigma$ to prove statement $T$; get as output Proof, a NIZK proof of knowledge of $r, c o m, s i g_{c o m}$ such that $T$ is true; send $c$, Proof to $U_{2}$.

- $\mathbf{U}_{2}$ : use algorithm $\mathrm{V}_{2}$, statement $T$ and $\sigma$ to verify Proof; if all the verifications are successful then accept the coin $c$.

Observe that $U_{1}$ cannot directly pass $s i g_{\text {com }}$ to $U_{2}$, for otherwise the coin $c$ would be later easily traceable from the Bank. On the other hand, he can prove the knowledge of a valid commitment com of some coin $c$ and a valid signature sig $_{\text {com }}$ for the commitment.

To transfer a coin $c$ to another user $U_{3}$, user $U_{2}$ simply sends him the coin $c$ and the string Proof received by $U_{1}$.

The protocol to deposit a coin is the same than that for spending a coin, where a user $U$ plays the role of the spender and the Bank that of the receiver.

The technique of signing a commitment in the protocol for withdrawing a coin and proving the knowledge of such a signature in the protocol for spending a coin has been used also in [9] to obtain an anonymous version of the NIZK proof system of knowledge with preprocessing given in [6].

\section{Allowing dividability of coins}

In this section we show how using the general paradigm for digital money described in the previous section and the cryptographic tools of pseudo-random functions and secure commitment schemes, it is possible to obtain the property of dividability of coins. This property has been first given in [20], using quadratic residues.

Let $m$ be the (constant) number of different values that a coin can assume; that is, the value of a coin will be $2^{k}$, for some $k \in\{0, \ldots, m-1\}$. Also, let $F=\left\{f_{s},|s|=n\right\}$ a collection of pseudo-random functions, written on the public file PF.

Consider the protocol for withdrawing a coin: the Bank has to issue coins in such a way that at a later stage it will be possible for a user $U_{1}$ to divide the coin withdrawn in smaller fractions and thus to spend an arbitrary piece of this coin to a certain user $U_{2}$. Moreover, the user $U_{2}$ shall be able to do the same with the coin received.

To this end, we require that each owner $U_{1}$ of a coin $c$ of value $2^{k}$, for some $k \in\{0, \ldots, m-1\}$, can compute two coins $c_{1}$ and $c_{2}$ of value $2^{k-1}$, and so on recursively. Thus, to the coin $c$ one can associate a complete binary tree $T_{c, k}$ of height $k$ in which each node is associated to a coin: that is, the root is associated to $c$, each of the 2 nodes at level 1 is associated to a coin of value $2^{k-1}$, and each 
of the $2^{h}$ nodes at level $h$ is associated to a coin of value $2^{k-h}$, for $h=2, \ldots, k$. In order to reach our goal, the tree $T_{c, k}$ has to satisfy the following properties: a) before the execution of the spending protocol, $U_{1}$ is able to compute any coin he desires in the tree whose root is associated to $c$ (this allows user $U_{1}$ to divide a coin owned into smaller fractions and thus pass any piece of it to a user $U_{2}$ ), and b) after $U_{1}$ has given a coin $d$ at level $k$ to $U_{2}, U_{2}$ is able to compute any coin he desires in the subtree whose root is associated to $d$ (this allows $U_{2}$ to do the same with the coin received).

Let us informally describe how the protocol for withdrawing a coin is modified in order to obtain the property of dividability of coins. In order to withdraw a coin of value $2^{k}$, a user $U_{1}$ randomly chooses $c \in\{0,1\}^{n}$ and computes $e_{i}=$ $f_{s_{2}}(c), r_{i}=f_{s_{1}}(\sigma), c_{i}=e_{i} \circ s_{2 i} \circ s_{2 i+1}$ and $d_{i}=E\left(c_{i}, \sigma, r_{i}\right)$, where the $s_{i}$ 's are randomly chosen so that $s_{i}=s_{2 i} \oplus s_{2 i+1}$, for $i=1, \ldots, 2^{k}-1$. Then $U_{1}$ sends to the Bank a $\left(2^{k+1}-1\right)$-tuple whose components represent the nodes of the complete binary tree $T_{c, k}$ of height $k$. Each node $i$ at level $h$ of $T_{c, k}$ is associated to a commitment $d_{i}$ to the coin $c_{i}$ of value $2^{k-h}$ that is represented by the concatenation of the following strings: $e_{i}$ and two strings $s_{2 i}, s_{2 i+1}$ that allow to decommit $d_{2 i}, d_{2 i+1}$ respectively. In such a way, for each coin $c$, the associated tree $T_{c, k}$ allows to compute all possible subdivisions of $c$ into coins of smaller values. Then, for each $i=1, \ldots, 2^{k+1}-1, U_{1}$ receives from the Bank a signature sig $_{d_{i}}$ of the commitment $d_{i}$, the index $i$ and the value $v_{i}$ of the coin $c_{i}$, where $s i g_{d_{z}}$ is computed using the scheme $\left(G_{B}, S_{B}, V_{B}\right)$. The knowledge of $s i g_{d_{z}}$ will represent the authorization from the Bank to spend the coin $c_{i}$ of value $v_{i}$.

In our scheme, given the random string $r_{1}, U_{i}$ can compute the random strings $r_{i}$ used for the commitment at each node $i$ of the tree $T_{c, k}$, and thus obtain all the coins that are possible subdivisions of the coin $c$ requested. Moreover, as we will see later, to spend a part of $c$, say of value $2^{k-h}$, a second user $U_{2}$ gives only the random string used for a commitment at a node at level $h$. It only remains to describe how a user computes the random strings $r_{i}$ at each node $i$ of a tree using the random string $r_{1}$ associated to the root. The commitment $d_{i}$ in node $i$ of the tree $T_{c, k}$ is computed using as random string $r_{i}=f_{s_{i}}(\sigma)$, where $\sigma$ is the random reference string written on the public file $\mathrm{PF}, f_{s}$, is a pseudo-random function and $s_{i}$ is a random string committed in the node $i$. Then, given $s_{i}$, a user can compute $r_{i}$, decommit the node $i$, and compute the indices $s_{2 i}, s_{2 i+1}$ and the random strings $r_{2 i}, r_{2 i+1}$. Thus, knowing a coin $c_{i}$ at a node $i$ allows to compute coins $c_{2 i}$ and $c_{2 i+1}$ at its two children. On the other hand, knowing a coin $c_{2 i}$ at a node $2 i$ does not allow to compute the coin $c_{i}$ at node $i$.

Now we formally describe the protocol for withdrawing a coin of value $2^{k}$.

\section{Withdrawing a coin:}

- U: randomly choose two $n$-bit strings $c, s_{1}$;

for $i=1, \ldots, 2^{k}-1$,

randomly choose the $n$-bit strings $s_{2 i}$ and compute $s_{2 i+1}=s_{i} \oplus s_{2 i}$;

for $i=1, \ldots, 2^{k+1}-1$, 
compute $e_{i}=f_{s_{i}}(c), r_{i}=f_{s_{i}}(\sigma), c_{i}=e_{i} \circ s_{2 i} \circ s_{2 i+1}$ and $d_{i}=E\left(c_{i}, \sigma, r_{i}\right)$; send the tree $T_{c, k}=\left(d_{1}, \ldots, d_{2^{k+1}-1}\right)$ to the Bank.

- Bank: for $i=1, \ldots, 2^{k+1}-1$,

let $v_{i}$ be the value of coin $d_{i}$;

compute $s i g_{d_{i}}=S_{B}\left(s k_{B}, d_{i} \circ i \circ v_{i}\right)$ and send $s i g_{d_{z}}$ to $U$.

- U: for $i=1, \ldots, 2^{k+1}-1$,

if $V_{B}\left(p k_{B}, d_{i} \circ i \circ v_{i}, s i g_{d_{i}}\right)=1$ then accept the coin $c_{i}$.

To deal with the case of dividability of coins, the spending protocol is modified in the following way. A user $\mathrm{U}_{1}$ has received an electronic coin $c$ of value $2^{k}$ from another user (or from the Bank) and wants to give a coin $c_{i}$ of value $2^{h}$ to user $U_{2}$. User $U_{1}$ sends the string $s_{i}$, the commitment $d_{i}$ and proof $f_{i}$, a NIZK proof of knowledge of $c_{i}$, of a signature of $i, v_{i}$ and $d_{i}$. Also, user $U_{1}$ sends to user $U_{2}$ all the strings $s_{l}, d_{l}$, proof $f_{l}$ that are associated to nodes in the subtree rooted at $c_{i}$. If all the proofs are convincing, then user $U_{2}$ accepts the coin $c_{i}$ from $U_{1}$. Formally, the protocol is:

\section{Spending a coin:}

- $\mathbf{U}_{1}$ : send $c$ to $\mathrm{U}_{2}$;

for $j \in\left\{1, \ldots, 2^{m}-1\right\}$ such that $c_{j}$ is associated to a descendant of $c_{i}$, let $T_{j}$ be the statement "there exist $r_{j}$, sig $g_{d_{j}}$ such that

$d_{j}=E\left(f_{s_{j}}(c) \circ s_{2 j} \circ s_{2 j+1}, \sigma, r_{j}\right), r_{j}=f_{s_{1}}(c)$

and $V_{B}\left(p k_{B}, d_{j} \circ j \circ v_{j}, s i g_{d_{3}}\right)=1 "$;

use algorithm $\mathrm{P}_{2}$, inputs $r_{j}$, sig $g_{d_{j}}$, and $\sigma$ to prove statement $T_{j}$;

get as output $P$ roof $f_{j}$, a NIZK proof of knowledge of $r_{j}, s i g_{d_{j}}$

such that $T_{j}$ is true;

send $s_{j}, d_{j}$, Proof $_{j}$ to $U_{2}$.

- $\mathbf{U}_{2}$ : for $j \in\left\{1, \ldots, 2^{m}-1\right\}$ such that $c_{j}$ is associated to a descendant of $c_{i}$,

use algorithm $\mathrm{V}_{2}$, statement $T_{j}$ and $\sigma$ to verify $\operatorname{Proof}_{j}$;

if all the verifications are successful then accept the coin $c$.

The protocol to deposit a coin is similar to that for spending a coin, where a user $U$ plays the role of the spender and the Bank that of the receiver. The only difference is that, instead of directly sending the commitment $d_{i}$, user $U$ sends a non-interactive zero-knowledge proof of knowledge of $d_{i}$, so that the Bank cannot use $d_{i}$ to trace coin $c_{i}$ to the user which had originally withdrawn it.

\section{Avoiding multiple spending of coins}

In this section we consider the problem of avoiding multiple spending of coins in the general paradigm for digital money described in the previous sections.

First of all we see the case in which dividability of coins is not allowed. To avoid the double spending of a same coin, while spending a coin, a user should give 
another message such that one of these messages gives no significant information on the spender, but two of these messages for a same coin give sufficient information to determine the author of the double spender. Nice solutions to this problem have been given in $[6,13,12]$. As it is possible to use these ideas for our general paradigm, we immediately get a way for avoiding double spending in our setting by a simple application of results in the cited papers. However, these ideas seem difficult to extend to the case of transferability of coins. Thus, we give a different technique based on signatures which, even if working under complexity assumptions (this is not the case for the cited techniques), allows to determine the author of a double spending also in the case in which the coin is transferred to many users before being deposited to the Bank. We observe that we have a sequence of spending protocols followed by a deposit protocol, and the Bank has to realize that a double spending has occurred only after the deposit protocol. Thus, to allow the Bank to discover the author of such a fraud, it must be the case that the user playing the role of spender of a certain coin has to commit to this action in some sense. This can be done in the following way: while spending a coin $c$, user $U_{1}$ also sends a signature of the coin and of the identity of the receiver of that coin. This signature can be seen as a commitment to the fact that user $U_{1}$ is passing the coin $c$ to user $U_{2}$.

The modification to the protocols of previous sections are the following: while opening an account, each user $U$ generates a pair $\left(p k_{U}, s k_{U}\right)$ for his signature scheme $\left(G_{U}, S_{U}, V_{U}\right)$ and writes $p k_{U}$ on the public file PF. Then, while passing coin $c$ to user $U_{2}$, user $U_{1}$ computes the signature $d s i g_{U_{1}, U_{2}, c}=S_{U_{1}}\left(s k_{U_{1}}, c \circ\right.$ $I D_{U_{2}}$ ) and sends it to $U_{2}$. In this way, the Bank realizes that a double spending has occurred as she receives two signatures from different users of a same coin. Then, in order to discover the author of a double-spending, the bank runs the procedure Detect. In this procedure the Bank broadcasts a message stating that a double-spending of coin $c$ has occurred (for example using the public file). To prove this, the Bank writes on the public file the two different signatures dsig., of a same coin $c$. At this point each user $U_{j}$ that has received coin $c$ in some

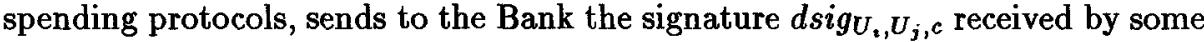
user $U_{i}$ with this coin, thus proving that he has received the coin $c$ by user $U_{i}$. In this way each user involved in this phase will reveal an identity of another user who has spent coin $c$ and thus the identity of the author of the double-spending will be revealed twice to the Bank. More precisely, the procedure Detect is the following:

Procedure Detect $\left(c, d_{s i g_{1}}, d_{s i g_{2}}\right)$.

- Bank: Broadcast a message in which it is stated that a double-spending has occurred and users who have received the coin $c$ in some spending protocol have to prove their honesty; write on the public file $c, d s i g_{1}, d s i g_{2}$.

- Each user $\mathbf{U}_{j}$ : If he has received the coin $c$ in some spending protocol then send the signature $d s i g$ received with that coin. 
- Bank: verify that the signatures received are properly computed by running the corresponding verification algorithms; also, reconstruct the complete history of the coin $c$; if a user $U$ sends a message not properly computed or the identity of some user $U$ is received from two different users then $U$ is the author of a double spending.

Let us now consider the case in which dividability of coins is allowed. In this case a dishonest $U$ could spend too many coins contained in the tree $T_{c, k}$, that is a set of coins for which the sum of values is greater than $2^{k}$. To avoid this, we impose that once a user has spent a coin $c_{i}$ in the tree $T_{c, k}$ he cannot spend coins $c_{j}$ in $T_{c, k}$ such that $c_{i}$ and $c_{j}$ belong to a same path starting from the root and finishing to a leave of $T_{c, k}$. Thus we say that a coin $c_{i}$ is spendable by user $U_{1}$ if all paths from the root to any leave of $T_{c, k}$ and containing $c_{i}$ do not contain coins that have already been spent by $U_{1}$. Also, we call inconsistent two coins associated to nodes in a same path from the root to a leave of a tree $T_{c, k}$.

Now, let us consider the spending protocol. Again, a user $U_{1}$ has received an electronic coin $c$ of value $2^{k}$ from another user (or from the Bank) and wants to give a coin $c_{i}$ of value $2^{h}$ to user $U_{2}$. The protocol is modified in the following way: first of all user $\mathrm{U}_{1}$ chooses $c_{i}$ as a spendable coin; then, while passing coin

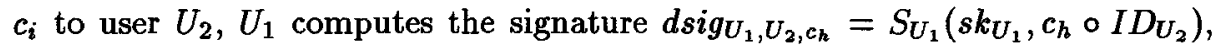
for each $c_{h}$ in the subtree rooted at $c_{i}$, and sends it to $U_{2}$. Thus, a user spending correctly two inconsistent coins will pass two different signatures of a same coin, and so, also in this case, the Bank realizes that a double spending has occurred as she receives two different signatures of a same coin. In order to determine the author of such a double spending, the Bank proceeds exactly as before.

Remarks. Our solution allows transferability of coins without giving any increase in the size of the coins transferred. To obtain this result, our protocol cannot satisfy anonymous spending; that is, $U_{1}$ 's identity can be computed form the message sent by $U_{1}$ to $U_{2}$ in the protocol for spending a coin. On the other hand, if anonymous spending is required, the main result of [5] states that transferred coins grow in size. The time users have to keep the signatures received in the spending protocols depends from the implementation: for istance, if it is required that each coin must be deposited before the end of the day in which it has been issued, users keep their signatures for at most one day.

\section{Proofs and properties}

By properly putting together the procedures written in Sections $4,5,6$, it is easy to construct the fourtuple $\mathcal{Q}=($ Open, WithDraW, SPEND, Deposit). Also, let $\mathcal{S}=\left\{B a n k, U_{1}, \ldots, U_{n}\right\}$. In this section we give a sketch of proof that the pair $(\mathcal{S}, \mathcal{Q})$ is an off-line electronic cash system and see that the electronic cash system given satisfies also the properties of dividability and spending of pieces of coins and off-line spending of coins. First, we show that the four requirements of Definition 2 are satisfied. 
No forging: Suppose that given the transcripts of $k$ protocols $\mathrm{W}_{1}, \ldots, \mathrm{W}_{k}$, there exists an efficient algorithm $A d v$ which computes $k+1$ coins $c_{1}, \ldots, c_{k+1}$ such that with nonnegligible probability for each $i=1, \ldots, k+1, \mathbf{S}=\left(\operatorname{Adv}, \mathbf{S}_{2}\right)\left(c_{i}, \cdot\right)=$ $(\cdot, a c c e p t)$. Then the algorithm Adv can be used to efficiently compute a signature of a given message $m$ without the knowledge of the secret key $s k_{B}$ in the following way. On input $m$, generate coins $c_{1}, \ldots, c_{k}$ and run $S=\left(A d v, S_{2}\right)$ on input $\left(c_{i}, \cdot\right)$ for $i=1, \ldots, k+1$, where $c_{k+1}=m$. The transcript of $\mathrm{S}=\left(A d v, \mathrm{~S}_{2}\right)$ on input $\left(c_{k+1}, \cdot\right)$ is a valid proof of knowledge of the signature of $m$. This can be used to contradict the properties of the proof system of knowledge $(\mathrm{P}, \mathrm{V})$ or of the signature scheme $\left(G_{B}, S_{B}, V_{B}\right)$.

No tracing: Suppose that given the transcripts of $k$ protocols $\mathrm{W}_{1}, \ldots, \mathrm{W}_{k}$ and of $k$ protocols $\mathrm{D}_{1}, \ldots, \mathrm{D}_{k}$, there exists an efficient algorithm $A d v$ which computes a coin $c$ such that with probability at least $1 / 2+$ a nonnegligible term, for some $i, j \in\{1, \ldots, n\}$ and $l \in\{i, j\}$, it holds that $\mathrm{D}=\left(\mathrm{D}_{1}, A d v\right)(c, \cdot)=(\cdot$, accept $)$ and $\mathrm{W}=\left(A d v, \mathrm{U}_{l}\right)(\cdot, c)=(\cdot$, accept $)$; or $\mathrm{D}=\left(\mathrm{D}_{1}, A d v\right)(c, \cdot)=(\cdot$, accept $)$ and $\mathrm{S}=\left(\mathrm{U}_{l}, \cdot\right)\left(c_{i}, \cdot\right)=(\cdot$, accept $)$. Then the algorithm $A d v$ can be used to efficiently compute with nonnegligible probability the identity of the withdrawer in some withdrawing protocol or that of the spender in some spending protocol of coin c. This contradicts the properties of the non-interactive zero-knowledge proof system of knowledge used.

No double spending: Suppose that there exists an efficient nonuniform algorithm $\overline{A d v}$ which computes a pair $\left(c_{1}, c_{2}\right)$ of coins such that $\mathrm{S}=\left(\operatorname{Adv}, \mathrm{S}_{i}\right)\left(c_{i}, \cdot\right)=$ $(\cdot$, accept $)$ for $i=1,2$. Then, a same coin is spent twice, and the Bank can realize that a double spending has occurred and identify the author of the double spending by running the procedure Detect described in the previous section. In fact, if a double spending has occurred, then a user $U$ (by running algorithm $A d v$ ) has given a same coin $c$, respectively to a user $V_{1}$ and another user $Z_{1}$. Now, suppose that $V_{1}$ has given $c$ to $V_{2}$, and so on until some $V_{h}$ has given it to the Bank. Analogously, suppose that $Z_{1}$ has given the same coin to $Z_{2}$, and so on until some $Z_{k}$ has given it to the Bank (where the $V_{i}$ and the $Z_{j}$ are not necessarily different). When running the procedure Detect, the Bank uses the signatures received to completely reconstruct the history of the coin $c$. In particular, she computes the two directed paths $V_{h}, V_{h-1}, \ldots, V_{1}, U$ and $Z_{k}, Z_{k-1}, \ldots, Z_{1}, U$ that have been taken (in the opposite direction) by the coin $c$ during the spending protocols. Thus she recognizes the author of the double-spending $U$ from the fact that he is the source of these two paths.

No framing: Suppose that given the transcripts of $k$ protocols $\mathrm{W}_{1}, \ldots, \mathrm{W}_{k}$ and of $k$ protocols $\mathrm{D}_{1}, \ldots, \mathrm{D}_{k}$, there exists an efficient algorithm $A d v$ which computes a coin $c$ such that for some $i, j \in\{1, \ldots, n\}$, computes $l \in\{i, j\}$ such that $\mathrm{S}=\left(\mathrm{U}_{l}, \mathrm{U}_{h}\right)(c, \cdot)=\left(\cdot, c_{1} \circ\right.$ accept $)$ and $\mathrm{S}=\left(\mathrm{U}_{l}, \mathrm{U}_{k}\right)(c, \cdot)=\left(\cdot, c_{2} \circ\right.$ accept $)$, for some $h, k \in\{1, \ldots, n\}$ with probability $1 / 2+$ a term nonnegligible in $n$, where $c_{1}$ and $c_{2}$ are the messages sent by $\mathrm{U}_{l}$ to $\mathrm{U}_{k}$ and $\mathrm{U}_{b}$ while spending coin $c$. Then the algorithm $A d v$ can be used to efficiently compute a signature of a given message $m$ without the knowledge of the secret key $s k_{U_{l}}$. This contradicts the 
properties of the signature scheme $\left(G_{U_{l}}, S_{U_{l}}, V_{U_{l}}\right)$.

Now we see that the electronic cash system given satisfies also the properties of dividability of coins, spending of pieces of coins and off-line spending of coins.

Dividability of coins and spending of pieces of coins: In our electronic cash system a coin $c_{i}$ of value $2^{h}$ is the concatenation of the following strings: a string $e_{i}$ computed as $f_{s_{2}}(c)$, where $f_{s_{1}}$ is a pseudo-random function whose index $s_{i}$ is known only to the owner of the coin $c_{i}$; and two strings $s_{2 i}, s_{2 i+1}$. These last strings are the indices of the pseudo-random functions that generate the random string $r_{j}=f_{s_{j}}(\sigma)$, for $j=2 i, 2 i+1$ used to compute the commitments $d_{2 i}, d_{2 i+1}$ respectively. Thus, given $s_{2 i}, s_{2 i+1}$, the owner of $c_{i}$ can decommit $d_{2 i}, d_{2 i+1}$ and compute the two coins $c_{2 i}, c_{2 i+1}$ of value $2^{h-1}$ in which $c_{i}$ can be divided. By repeating this process, given a coin $c$ of value $2^{k}$, any user $U_{i}$ can obtain any coin of value $2^{k-h}$ as value, for $h=1, \ldots, k$, and thus spend any coin of value $i$, for $i=1, \ldots, 2^{k}$.

Off-line spending of coins: This property is immediately obtained thanks to the non-interactive proof used in the spending protocol of a coin.

Acknowledgements. Many thanks go to Alfredo De Santis, Tatsuaki Okamoto, Giuseppe Persiano and Moti Yung for helpful discussions.

\section{References}

1. M. Blum, A. De Santis, S. Micali, and G. Persiano, Non-Interactive ZeroKnowledge, SIAM Journal of Computing, vol. 20, no. 6, Dec 1991, pp. 1084-1118.

2. M. Blum, P. Feldman, and S. Micali, Non-Interactive Zero-Knowledge and Applications, Proceedings of the 20th ACM Symposium on Theory of Computing, 1988, pp. 103-112.

3. S. Brands, Untraceable Off-line Cash in Wallets with Observers, in "Advances in Cryptology - CRYPTO 93", vol. 773 of "Lecture Notes in Computer Science”, Springer-Verlag, pp. 302-318.

4. D. Chaum, A. Fiat, and M. Naor, Untraceable Electronic Cash, in "Advances in Cryptology - CRYPTO 88", vol. 403 of "Lecture Notes in Computer Science", Springer-Verlag, pp. 319-327.

5. D. Chaum and T. Pedersen, Transferred Cash Grows in Size, in "Advances in Cryptology - Eurocrypt 92", vol. 658 of "Lecture Notes in Computer Science", Springer-Verlag, pp. 390-407.

6. A. De Santis and G. Persiano, Communication Efficient Zero-Knowledge Proof of knowledge (with Application to Electronic Cash), in Proceedings of STACS 92, pp. 449-460.

7. A. De Santis and G. Persiano, Zero-Knowledge Proofs of Knowledge Without Interaction, Proceedings of the 33rd IEEE Symposium on Foundations of Computer Science, 1992, pp. 427-436.

8. G. Di Crescenzo, A Non-Interactive Electronic Cash System, in Proceedings of Italian Conference on Algorithms and Complexity (CIAC 94), Springer Verlag. 
9. G. Di Crescenzo, Anonymous NIZK Proofs of Knowledge with Preprocessing, manuscript.

10. W. Diffie and M. E. Hellman, New Directions in Cryptography, IEEE Transaction on Information Theory, vol. IT-22, no. 6, Nov. 1976. pp.644-654.

11. U. Feige, A. Fiat, and A. Shamir, Zero-knowledge Proofs of Identity, Journal of Cryptology, vol. 1, 1988, pp. 77-94.

12. N. Ferguson, Single Term Off-Line Coins, in "Advances in Cryptology - Eurocrypt 93", vol. 765 of "Lecture Notes in Computer Science", Springer-Verlag, pp. 318328.

13. M. Franklin and M. Yung, Secure and Efficient Off-Line Digital Money, in Proceedings of ICALP 93, vol. 700 of "Lecture Notes in Computer Science", SpringerVerlag, pp. 265-276.

14. O. Goldreich, S. Goldwasser, and S. Micali, How to Construct Random Functions, Journal of the Association for Computing Machinery, vol. 33, no. 4, 1986, pp. $792-807$.

15. S. Goldwasser, S. Micali, and C. Rackoff, The Knowledge Complexity of Interactive Proof-Systems, SIAM Journal on Computing, vol. 18, n. 1, February 1989.

16. S. Goldwasser, S. Micali, and R. Rivest, A Digital Signature Scheme Secure Against Adaptive Chosen-Message Attack, SIAM Journal of Computing, vol. 17, n. 2, April 1988, pp. 281-308.

17. S. Goldwasser and R. Ostrovsky, Invariant Signatures and Non-Interactive ZeroKnowledge Proofs are Equivalent, in "Advances in Cryptology - CRYPTO 92", vol. 470 of "Lecture Notes in Computer Science", Springer-Verlag, pp. 246-259.

18. M. Naor, Bit Commitment using Pseudo-randomness, in "Advances in Cryptology - CRYPTO 89", vol. 435 of "Lecture Notes in Computer Science", Springer-Verlag.

19. M. Naor and M. Yung, Universal One-way Hash Functions and their Cryptographic Applications, Proceedings of 21st ACM Symposium on the Theory of Computing, 1989.

20. T. Okamoto and K. Ohta, Universal Electronic Cash, in "Advances in Cryptology - CRYPTO 91", vol. 576 of "Lecture Notes in Computer Science", Springer-Verlag, pp. 324-337.

21. T. Okamoto and K. Ohta, Disposable Zero-knowledge Authentications and their Applications to Untraceable Electronic Cash, in "Advances in Cryptology - CRYPTO 89", vol. 435 of "Lecture Notes in Computer Science", Springer-Verlag, pp. 481496.

22. J. Rompel, One-way Functions are Necessary and Sufficient for Secure Signatures, Proceedings of the 22nd ACM Symposium on Theory of Computing, 1990, pp. 387-394.

23. M. Tompa and H. Woll, Random Self-Reducibility and Zero-knowledge Interactive Proofs of Possession of Information, Proceedings of 28th Symposium on Foundations of Computer Science, 1987, pp. 472-482. 